\title{
Correction to: CT iterative vs deep learning reconstruction: comparison of noise and sharpness
}

\author{
Chankue Park $^{1} \cdot$ Ki Seok Choo ${ }^{1}$ (D) $\cdot$ Yunsub Jung ${ }^{2} \cdot$ Hee Seok Jeong ${ }^{1} \cdot$ Jae-Yeon Hwang ${ }^{1} \cdot$ Mi Sook Yun ${ }^{3}$ \\ Published online: 16 December 2020 \\ (C) European Society of Radiology 2020, corrected publication 2021
}

\section{Correction to: European Radiology}

https://doi.org/10.1007/s00330-020-07358-8

The original version of this article, published on 15 October 2020, unfortunately contained mistakes. The following corrections have therefore been made in the original:

The heading "Sharpness evaluation" should be a subheading of "Quantitative analysis", the presentation of Table 1 was incorrect and affiliation 1 was incomplete.

The original article has been corrected.

Publisher's note Springer Nature remains neutral with regard to jurisdictional claims in published maps and institutional affiliations.

The online version of the original article can be found at https://doi.org/ $10.1007 / \mathrm{s} 00330-020-07358-8$

Ki Seok Choo

kschoo0618@naver.com

1 Department of Radiology, Research Institute for Convergence of Biomedical Science and Technology, Pusan National University Yangsan Hospital, Yangsan, South Korea

2 CT Research Team, GE Healthcare Korea, Seoul, South Korea

3 Division of Biostatistics, Pusan National University Yangsan Hospital, Yangsan, South Korea 
Table 1 Quantitative analysis (conventional method)

\begin{tabular}{|c|c|c|c|c|c|c|}
\hline & AV-80 & AV-100 & TF-L & TF-M & TF-H & $p^{*}$ \\
\hline \multicolumn{7}{|l|}{ Aorta } \\
\hline $\mathrm{HU}$ & $403.1^{\mathrm{b}} \pm 172.0$ & $401.0^{\mathrm{a}} \pm 171.1$ & $406.3^{c} \pm 171.8$ & $406.3^{c} \pm 171.7$ & $406.3^{c} \pm 171.8$ & $<0.001$ \\
\hline SD & $27.9^{\mathrm{c}} \pm 10.1$ & $26.5^{\mathrm{b}} \pm 11.1$ & $32.5^{\mathrm{d}} \pm 8.0$ & $28.6^{\mathrm{c}} \pm 8.7$ & $24.6^{\mathrm{a}} \pm 9.6$ & $<0.001$ \\
\hline SNR & $14.9^{\mathrm{b}} \pm 5.2$ & $16.0^{c} \pm 5.7$ & $12.6^{\mathrm{a}} \pm 4.6$ & $14.5^{\mathrm{b}} \pm 5.2$ & $17.3^{c} \pm 6.4$ & $<0.001$ \\
\hline $\mathrm{CNR}$ & $20.3^{b} \pm 6.0$ & $21.8^{c} \pm 6.7$ & $17.0^{\mathrm{a}} \pm 5.1$ & $19.6^{\mathrm{b}} \pm 5.9$ & $23.5^{\mathrm{c}} \pm 7.6$ & $<0.001$ \\
\hline \multicolumn{7}{|c|}{ Femoral artery } \\
\hline $\mathrm{HU}$ & $442.6^{\mathrm{b}} \pm 179.0$ & $436.6^{\mathrm{a}} \pm 176.6$ & $450.0^{\mathrm{c}} \pm 181.1$ & $450.6^{\mathrm{c}} \pm 180.6$ & $450.5^{\mathrm{c}} \pm 180.9$ & $<0.001$ \\
\hline SD & $46.6^{\mathrm{a}, \mathrm{b}, \mathrm{c}} \pm 40.7$ & $45.7^{\mathrm{a}, \mathrm{b}, \mathrm{c}} \pm 40.9$ & $47.9^{c} \pm 41.9$ & $46.1^{\mathrm{b}} \pm 42.5$ & $44.5^{\mathrm{a}} \pm 43.2$ & 0.007 \\
\hline SNR & $12.7^{\mathrm{a}, \mathrm{b}} \pm 6.4$ & $13.0^{\mathrm{a}, \mathrm{b}, \mathrm{c}} \pm 6.6$ & $11.7^{\mathrm{a}} \pm 5.1$ & $12.7^{\mathrm{b}} \pm 5.7$ & $13.9^{c} \pm 6.7$ & 0.001 \\
\hline $\mathrm{CNR}$ & $17.4^{\mathrm{a}, \mathrm{b}, \mathrm{c}} \pm 9.0$ & $17.6^{\mathrm{c}, \mathrm{d}} \pm 8.6$ & $15.7^{\mathrm{a}} \pm 6.2$ & $17.0^{\mathrm{b}, \mathrm{c}} \pm 7.2$ & $18.8^{\mathrm{d}} \pm 8.9$ & 0.001 \\
\hline \multicolumn{7}{|c|}{ Popliteal artery } \\
\hline HU & $473.1^{\mathrm{b}} \pm 177.3$ & $466.7^{\mathrm{a}} \pm 175.4$ & $488.1^{\mathrm{e}} \pm 178.6$ & $487.7^{\mathrm{d}} \pm 178.8$ & $487.1^{\mathrm{c}} \pm 179.0$ & $<0.001$ \\
\hline SD & $67.7^{\mathrm{a}} \pm 39.7$ & $67.2^{\mathrm{a}} \pm 38.7$ & $70.4^{b} \pm 40.1$ & $70.1^{\mathrm{b}} \pm 40.5$ & $70.0^{\mathrm{b}} \pm 41.0$ & $<0.001$ \\
\hline SNR & $9.1^{\mathrm{a}} \pm 6.0$ & $9.2^{\mathrm{a}} \pm 7.1$ & $8.6^{\mathrm{a}} \pm 4.8$ & $8.8^{\mathrm{a}} \pm 5.1$ & $8.9^{\mathrm{a}} \pm 5.4$ & 0.390 \\
\hline CNR & $12.2^{\mathrm{a}} \pm 8.3$ & $12.4^{\mathrm{a}} \pm 10.2$ & $11.2^{\mathrm{a}} \pm 6.0$ & $11.4^{\mathrm{a}} \pm 6.4$ & $11.7^{\mathrm{a}} \pm 7.1$ & 0.276 \\
\hline \multicolumn{7}{|l|}{ Liver } \\
\hline HU & $140.5^{\mathrm{a}} \pm 23.1$ & $140.6^{b} \pm 23.1$ & $141.0^{\mathrm{c}} \pm 23.1$ & $141.1^{\mathrm{c}} \pm 23.1$ & $141.1^{\mathrm{c}} \pm 23.0$ & $<0.001$ \\
\hline SD & $25.4^{\mathrm{b}} \pm 5.6$ & $22.7^{\mathrm{a}} \pm 5.8$ & $30.6^{\mathrm{d}} \pm 4.7$ & $26.8^{\mathrm{c}} \pm 5.2$ & $23.1^{\mathrm{a}} \pm 5.9$ & $<0.001$ \\
\hline SNR & $5.8^{\mathrm{c}} \pm 1.6$ & $6.6^{\mathrm{d}} \pm 2.2$ & $4.7^{\mathrm{a}} \pm 1.0$ & $5.5^{\mathrm{b}} \pm 1.3$ & $6.5^{\mathrm{d}} \pm 2.0$ & $<0.001$ \\
\hline $\mathrm{CNR}$ & $8.4^{\mathrm{c}} \pm 2.3$ & $9.5^{\mathrm{d}} \pm 3.0$ & $6.9^{\mathrm{a}} \pm 1.5$ & $7.9^{\mathrm{b}} \pm 2.0$ & $9.4^{\mathrm{d}} \pm 2.9$ & $<0.001$ \\
\hline \multicolumn{7}{|c|}{ Psoas muscle } \\
\hline HU & $66.1^{\mathrm{a}} \pm 7.6$ & $66.0^{\mathrm{a}} \pm 7.5$ & $67.4^{b} \pm 7.9$ & $67.2^{b} \pm 7.5$ & $67.2^{\mathrm{b}} \pm 7.5$ & $<0.001$ \\
\hline SD & $17.4^{\mathrm{b}} \pm 4.4$ & $14.6^{\mathrm{a}} \pm 4.7$ & $24.3^{\mathrm{d}} \pm 3.7$ & $19.8^{\mathrm{c}} \pm 4.0$ & $15.1^{\mathrm{a}} \pm 4.5$ & $<0.001$ \\
\hline SNR & $4.0^{\mathrm{c}} \pm 1.0$ & $4.9^{\mathrm{d}} \pm 1.5$ & $2.8^{\mathrm{a}} \pm 0.5$ & $3.5^{\mathrm{b}} \pm 0.7$ & $4.8^{\mathrm{d}} \pm 1.2$ & $<0.001$ \\
\hline CNR & $12.3^{\mathrm{c}} \pm 3.4$ & $15.2^{\mathrm{d}} \pm 4.8$ & $8.6^{\mathrm{a}} \pm 1.7$ & $10.6^{\mathrm{b}} \pm 2.4$ & $14.4^{\mathrm{d}} \pm 3.8$ & $<0.001$ \\
\hline
\end{tabular}

Data are mean value \pm standard deviation. The superscripts represent the same group of the Bonferroni post hoc test (the alphabetical order indicates the order, starting from the lowest mean value). AV-80 and AV-100 = ASIR-V with a blending factor of $80 \%$ and $100 \%$, respectively; TF-L, TF-M, and $\mathrm{TFH}=$ TrueFidelity with low, medium, and high strength levels, respectively; $\mathrm{HU}=$ mean CT number, $\mathrm{SD}=$ image noise, $\mathrm{SNR}=$ target $\mathrm{HU} /$ target $\mathrm{SD}$, and $\mathrm{CNR}=$ target $\mathrm{HU}$ - fat $\mathrm{HU} /$ target SD

* $p$ values were calculated with repeated measures ANOVA among the five groups 